\title{
Motivation as a Predictor of Drinking Outcomes After Residential Treatment Programs for Alcohol Dependence
}

\author{
Sarah Bauer, MSc, Werner Strik, MD, and Franz Moggi, PhD
}

\begin{abstract}
Objectives: Patients' motivation to change their substance use is usually viewed as a crucial component of successful treatment. The objective of this study was to examine whether motivation contributes to drinking outcomes after residential treatment for alcohol dependence.

Methods: Our sample included 415 Swiss patients from 12 residential alcohol treatment programs. We statistically controlled for important predictors, such as sex, employment, alcohol consumption before admission, severity of alcohol dependence, severity of psychiatric symptoms at admission, and alcohol-related self-efficacy at discharge. Abstinence, alcohol consumption, and time to first drink were used as primary outcome measures and were assessed 1 year after discharge from treatment.

Results: Action-oriented motivation to change substance use had a modest impact on drinking outcomes. At the 1-year follow-up, only the Taking Steps subscale of the Stages of Change Readiness and Treatment Eagerness Scale and alcohol-related self-efficacy were found to be significant predictors of abstinence and the number of standard drinks.

Conclusions: The impact of action-oriented motivation at admission to residential treatment is modest but still relevant, compared with other outcome predictors. It may be useful to focus treatment on improving action-oriented motivation to reduce substance use.
\end{abstract}

Key Words: 1-year drinking outcomes, alcohol dependence, residential treatment, SOCRATES, treatment motivation

( $J$ Addict Med 2014;8: 137-142)

A lcohol dependence is an important health problem that has serious biological, physiological, and social consequences and immense direct and indirect costs (Rehm, 2011). Reducing the health consequences of excessive alcohol consumption is therefore of wide interest in public health. Various studies

From the University Hospital of Psychiatry (SB, WS, FM), University of Bern, Bern, Switzerland; and Department of Psychology (FM), University of Fribourg, Fribourg, Switzerland.

Received for publication June 13, 2013; accepted November 21, 2013.

Supported by the Swiss National Science Foundation (grant no. 32-58803.99) and the University Hospital of Psychiatry, University of Bern.

The authors declare no conflicts of interest.

Send correspondence and reprint requests to Franz Moggi, $\mathrm{PhD}$, University Hospital of Psychiatry, University of Bern, Bolligenstrasse 111, 3000 Bern 60, Switzerland. E-mail: moggi@puk.unibe.ch.

Copyright (C) 2014 American Society of Addiction Medicine

ISSN: $1932-0620 / 14 / 0802-0137$

DOI: 10.1097/ADM.0000000000000013 have attempted to identify predictors of treatment outcomes in patients with alcohol dependence. Knowledge of such predictors provides 3 key benefits to clinicians. First, it is important to identify stable patient variables (eg, sex) that predict poor outcomes so that treatment may be adapted to focus on changing more malleable variables (eg, motivation for treatment). Second, such knowledge enables clinicians to identify target areas for treatment (eg, motivation). Finally, it contributes to prognosis accuracy (eg, improved counseling of patients and family) (Adamson et al., 2009). Because motivation to change substance use is viewed as a crucial component of treatment outcome, many recent studies have focused on its utility for predicting treatment outcomes. Most studies base their concept of motivation on the Transtheoretical Model of Change (TTM) proposed by Prochaska and DiClemente (1983). According to this model, 5 stages of change (precontemplation, contemplation, decision, action, and maintenance) represent different levels of readiness or motivation to change substance use.

The Stages of Change Readiness and Treatment Eagerness Scale (SOCRATES) (Miller and Tonnigan, 1996) is a questionnaire based on the TTM that has been widely used in important treatment efficacy studies (eg, Project MATCH, 1998). It measures readiness for change on 3 subscales: Recognition, Ambivalence, and Taking Steps. The Recognition subscale reflects the precontemplation and decision stages and refers to a person's capacity to recognize that his or her problems are due to drinking. The Ambivalence subscale reflects the contemplation stage and refers to a person's uncertainty about whether he or she wants to change his or her drinking behavior. The Taking Steps subscale includes items from the action and maintenance stages; a high score indicates that the person is already taking action to make a positive change in his or her drinking behavior (Miller and Tonnigan, 1996). Several studies have found a significant correlation between the stages of change measured by the SOCRATES and treatment outcomes (Project MATCH, 1998; Demmel et al., 2004; Bertholet et al., 2010; Hunter-Reel et al., 2010; Maisto et al., 2011; Small et al., 2012).

In a systematic review of 51 studies, Adamson et al. (2009) found that motivation was one of the most consistent predictors of alcohol treatment outcomes. In Project MATCH (1998), readiness to change and alcohol-related self-efficacy were the strongest predictors of outcomes. However, the predictive utility of the SOCRATES is often restricted to the Taking Steps subscale. Bertholet et al. (2010) showed that higher scores on this subscale were associated with better drinking 
outcomes (eg, less than 14 standard drinks per week or less than 4 drinks per occasion for men, and less than 7 drinks per week or less than 3 drinks per occasion for women). Demmel et al. (2004) showed that readiness to change accounted for $9.4 \%$ of the variance in treatment outcomes between abstainers and relapsers. Similarly, Maisto et al. (2011) found that the Taking Steps score was a good predictor of alcohol consumption at 6 and 12 months after treatment. Moreover, using structural equation modeling, Small et al. (2012) found significant direct paths between Taking Steps scores and reported drinks per drinking day and alcohol severity at 12 months after treatment.

However, results regarding the predictive utility of motivation are contradictory. Hewes and Janikowski (1998) found that subject groups based on levels of readiness to change did not differ from each other with respect to their composite scores (that included all 7 areas) on the Addiction Severity Index at a 1-month follow-up. Lemke and Moos (2003) and Heather and McCambridge (2013) found that initial motivation for treatment was not related to long-term outcomes measured at 12 months and 5 years after the beginning of treatment. A higher Recognition score was associated with higher rates of drinking in 2 studies (Bertholet et al., 2009; Small et al., 2012). Small et al. (2012) suggested that a high Recognition score may not be sufficient to change substance use without a similarly high Taking Steps score.

In their comprehensive review of predictors of treatment outcome, Adamson et al. (2009) noted that further research is needed on the most likely predictors, including motivation and other variables such as sex, employment, alcohol use before treatment, dependence severity, and psychiatric symptoms. They suggested focusing on these variables to understand which predictors of specific outcome measures are effective.

Hence, the objective of the current study was to examine the contribution of motivation to change substance use (as measured by the 3 SOCRATES subscales) on drinking outcomes in a large multicenter sample of patients attending residential alcohol treatment programs. Abstinence, alcohol consumption, and time to first drink were used as primary outcome measures and were assessed 1 year after discharge from residential treatment. In consideration of the comprehensive review by Adamson et al. (2009), and in contrast to many other studies, we statistically controlled for other important predictors, such as sex, employment, alcohol consumption before admission, severity of alcohol dependence, severity of psychiatric symptoms at admission, and alcohol-related self-efficacy at discharge. Alcohol-related self-efficacy was included because it has been shown to be a very important predictor of 1-year outcomes when assessed at discharge from treatment (Ludwig et al., 2013). Our goal is to better understand which subscales of the SOCRATES are effective predictors of which outcome measures.

\section{METHODS}

\section{Procedure}

At admission to a residential abstinence-oriented alcohol use disorder (AUD) treatment program, detoxified patients completed an intake information form (IIF) that assessed so- ciodemographic characteristics, such as age, sex, and employment; indices of substance use and its consequences; psychological and social functioning; the number of prior hospitalizations; and prior involvement in outpatient treatment and self-help activities. One year after discharge from the index stay (1-year follow-up), patients completed a follow-up information form (FIF) that assessed the same content areas as the IIF. The assessment instruments used in the IIF and FIF are described in greater detail in the Assessments section. The study was approved by the ethics committee of the Canton of Berne, Switzerland (proposal no.: 109/99).

\section{Patients}

All patients with substance use disorder (SUD) who entered into 1 of 12 residential treatment programs with an intended length of stay of 4 months (index stay) were asked to participate in this study. Of 1088 patients, 805 (74\%) agreed to participate and gave written informed consent. Of them, 587 patients had AUD without any other drug use disorder. Overall, $415(70.7 \%)$ patients completed the inventories at admission, discharge, and 1-year follow-up; 172 (29.3\%) patients did not send back the FIF despite attempts to reach them by followup letters and phone calls. Of those who did not complete the FIF, $22(12.8 \%)$ were deceased, $12(7.0 \%)$ explicitly stated that they did not want to participate anymore, $8(4.7 \%)$ changed residence without leaving a forwarding address, and $2(1.2 \%)$ committed suicide. No contact could be established with the remaining $128(74.3 \%)$ patients.

Patients who completed all measurements (respondents) were compared with nonresponding patients $(\mathrm{n}=172 ; 29.3 \%)$ on demographic variables, substance use, and psychological and social functioning characteristics. Respondents were slightly older than nonrespondents (46.7 vs 44.7 years, $t=$ $2.38, d f=585, P<0.05)$ and were more likely to be women $\left(77.0 \%\right.$ vs $\left.67.8 \%, \chi^{2}=4.74, d f=1, P<0.05\right)$, married (78.9\% vs $\left.67.2 \%, \chi^{2}=8.48, d f=1, P<0.01\right)$, and employed (75.7\% vs $\left.66.0 \%, \chi^{2}=6.11, d f=1, P<0.05\right)$. No other significant differences were found.

Approximately two thirds of patients were men and most were unmarried. Almost $60 \%$ of patients were employed when admitted for treatment, almost $80 \%$ had more than a high school education, and $40 \%$ had a comorbid psychiatric disorder. Percentages of predictor variables and means of the 3 motivation subscales are presented in Table 1 .

\section{Residential Treatment Programs for AUDs}

We selected 12 standard practice residential treatment programs for patients with AUD to capitalize on realistic treatment conditions and typical treatment orientations. In addition, we selected programs that were representative of the German-speaking part of Switzerland. Programs were financed by the canton and by obligatory public health insurance. For individuals who could not afford health insurance premiums, these premiums were paid by the community. Program staffing patterns depended on the number of patients but always included psychiatrists, psychologists, social workers, and nurses.

Program characteristics are described in greater detail and compared with residential AUD programs affiliated with 
TABLE 1. Patient Characteristics, Predictor Variables, and Mean Scores on the 3 Motivation Scales at Admission to the Index Residential Treatment

\begin{tabular}{lrr}
\hline Patient Characteristics & M/\% & SD \\
\hline Demographics & & \\
$\quad$ Age & 46.7 & 9.30 \\
Women & 34.0 & \\
Married & 39.8 & \\
Employed & 58.7 & \\
Education & & \\
\% less than high school & 13.0 & \\
\% high school & 8.5 & \\
\% high school + 2 y & 70.0 & \\
\% college & 8.5 & \\
Predictors at admission & & \\
Alcohol use 3 months before treatment & 17.0 & 10.69 \\
$\quad$ (the number of standard drinks) & & \\
Severity of alcohol dependence & 19.1 & 10.36 \\
Consequences of substance abuse & 14.4 & 8.53 \\
Severity of psychiatric symptoms & 23.6 & 15.21 \\
Treatment motivation (SOCRATES & & \\
$\quad$ subscales) & & \\
$\quad$ Recognition & 24.0 & 3.28 \\
$\quad$ Ambivalence & 8.3 & 3.43 \\
$\quad$ Taking Steps & 25.4 & 4.36 \\
Predictors at discharge & & \\
$\quad$ Presence of comorbid psychiatric disorder & 40.7 & \\
Alcohol-related self-efficacy & 7.8 & 1.85 \\
\hline
\end{tabular}

$n$ varies from 402 to 415 because of missing data.

SOCRATES, Stages of Change Readiness and Treatment Eagerness Scale.

the US Department of Veterans Affairs Health Care System in 2 publications by Moggi et al. (2007, 2010). Overall, Swiss programs are, on average, 5 times longer (122 days) than US programs (25 days), offer more individual and fewer group sessions, more clearly focus on substance abuse, are less 12step/Alcoholics Anonymous oriented, and place less emphasis on the disease model. Swiss and US programs are similarly oriented toward cognitive-behavioral treatment and place similar emphasis on the psychosocial model of SUDs.

\section{Assessments}

\section{Patient Characteristics at Treatment Admission}

Employment was coded as follows: $1=$ full or part-time employment and $0=$ not employed. Prior SUD treatment was measured by attendance in any inpatient or outpatient SUD treatment program 2 years before the index stay (coded as follows: $1=$ one or more, $0=$ none).

Fifteen items were used to assess patients' alcohol and drug use in the 3 months before treatment. Items were adapted from the Health and Daily Living Form (Moos et al., 1990) and the Treatment Outcome Prospective study (Hubard et al., 1989). Patients were asked about the frequency and quantity of beer, wine, and hard liquor they consumed, and about the frequency of use of various illicit drugs. Frequency was rated on a 5-point scale ( $0=$ "never," $4=$ "every day"), and quantity was assessed separately for each beverage in terms of cans of beer, glasses of wine, and shots/pints of hard liquor. Average pure alcohol use per day was calculated in grams and divided by $12.5 \mathrm{~g}$ to obtain the average daily number of standard drinks.
Severity of alcohol dependence was assessed by 9 items drawn from the Alcohol Dependence Scale, which was developed to correspond with the Diagnostic and Statistical Manual of Mental Disorders criteria for alcohol dependence (MaudeGriffin et al., 1992). Substance use problems were assessed with 15 items that included questions about job, family, and health problems (Maude-Griffin et al., 1992). The item responses for these 2 scales ranged from "never" (0) to "often" (4). The severity scores on the Alcohol Dependence Scale ranged from 0 to $36(\alpha=0.92)$ and the substance use problems scores ranged from 0 to $60(\alpha=0.80)$.

Psychiatric symptoms were assessed by selected items from the Brief Symptom Inventory, using the Depression, Anxiety, Paranoid Ideation, and Psychoticism scales (Derogatis, 1993). Items referred to the previous 3 months, and responses ranged from "not at all" (0) to "extremely" (4), with total scores ranging from 0 to 88 ( $\alpha=0.93)$.

Motivation for treatment was measured with an adapted version of the 19-item SOCRATES (Miller and Tonnigan, 1996) for residential treatment that had been used in a large US multisite study (Ouimette et al., 1997). This instrument differs slightly in wording from the original SOCRATES to better suit patients attending a residential treatment facility (Maude-Griffin et al., 1992). This version of the SOCRATES was translated into German and back-translated to English. When differences were identified between the 2 versions, items were revised and adapted accordingly. The SOCRATES measures readiness for change on 3 subscales: the Recognition subscale refers to the person's capacity to attribute his or her problems to drinking, the Ambivalence subscale reflects the person's uncertainty about his or her desire to change drinking behavior, and the Taking Steps subscale indicates that the person is already taking action to positively change his or her drinking behavior. Stages were assessed with items with responses ranging from "strongly disagree" (0) to "strongly agree" (4). The Recognition subscale consisted of 7 items, with a total score from 0 to 28 ( $\alpha=0.71$ ); the Ambivalence subscale had 4 items, with a total score ranging from 0 to $16(\alpha=0.46)$; and the Taking Steps subscale included 8 items, with a total score from 0 to $32(\alpha=0.71)$. Subscales were divided on the basis of results of the factor analysis by Miller and Tonnigan (1996), and the 3 subscale scores were calculated according to these authors' instruction. There was no overall difference in either the 3 subscales' means $(F=1.79-1.50, P \geq 0.05)$ or their variances (Levene test $=0.87-1.06, P \geq 0.05$ ) between participants from the 12 treatment programs.

\section{Patient Characteristics at Treatment Discharge}

Patients were provisionally diagnosed at admission, but definitive diagnoses based on the Tenth Revision of the International Classification of Diseases, guidelines (Dilling et al., 1991) were made at discharge by doctoral-level clinical treatment staff and included Axis I and Axis II disorders and medical conditions.

Alcohol-related self-efficacy was measured with the 14-item Situational Confidence Questionnaire (Miller et al., 1989), which measures the patient's ability to remain abstinent from alcohol in specific situations. For each item, patients 
indicate their level of confidence in remaining abstinent in a specified situation by choosing from a list of 6 options $(0 \%$, $20 \%, 40 \%, 60 \%, 80 \%$, and $100 \%$ ). For scoring purposes, percentages were converted to a 6-point scale that ranged from 0 (corresponding to $0 \%$ ) to 5 (corresponding to $100 \%$ ) for each item; these were then summed and divided by 14 to obtain a mean level of confidence in remaining abstinent $(\alpha=0.96)$.

\section{Outcome Measures}

We focused on the following outcome measures: $(a)$ abstinence from alcohol, assessed by whether patients reported any alcohol consumption (coded as $1=$ abstinent, and $0=$ not abstinent); (b) the number of standard drinks on typical drinking days, and (c) time to first alcohol use, defined as the number of days between the end of the index stay and consumption of the first alcoholic drink.

\section{Statistics}

To identify the most important predictors of alcohol use at 1-year follow-up, a stepwise logistic regression for abstinence was calculated for each SOCRATES subscale (Recognition, Ambivalence, and Taking Steps). Predictors (ie, sex, employment, alcohol consumption, severity of alcohol dependence, psychiatric symptoms, and alcohol-related selfefficacy) were entered in the first step, and the 3 SOCRATES subscales were entered in the second step. The same procedure was used for multiple regression analyses to test whether the 3 motivational subscales were associated with the number of standard drinks at 1-year follow-up. Abstinence was coded as 0 , to indicate that no standard drinks were consumed. Finally, for predictors that were significant in the regression models, Cox survival regressions were performed to predict the time to first drink after discharge from the residential treatment programs. For the Cox regressions, patients were divided into 2 groups for each SOCRATES scale, depending on whether they scored more or less than the mean. We conducted 1-way analysis of variance to test whether there were any differences in the means and variances of SOCRATES subscales between participating patients from the 12 treatment programs.

\section{RESULTS}

In the hierarchical logistic regression analysis, alcoholrelated self-efficacy and the Taking Steps subscale after controlling for self-efficacy were the only 2 significant predictors of abstinence at 1-year follow-up (Table 2). The Recognition subscale was not a significant predictor $(B=-0.059, P=$ 0.13 ), and although there was a trend for Ambivalence to be a predictor, it was not significant $(B=-0.056, P=0.08)$.

In the stepwise regression analysis, employment, alcohol-related self-efficacy, and Taking Steps after controlling for self-efficacy were shown to be significant predictors of the number of standard drinks on a typical drinking day at 1-year follow-up (Table 3). Again, neither Recognition $(B=$ $-0.006, P=0.91)$ nor Ambivalence $(B=-0.075, P=0.15)$ was a significant predictor.

The Cox survival regression analyses showed no significant differences in time to first drink between patients with high and low scores on the 3 SOCRATES subscales when all
TABLE 2. Results of the Hierarchical Logistic Regression Analysis With Abstinence as the Dependent Variable

\begin{tabular}{|c|c|c|c|c|c|}
\hline Predictors & $B$ & SE $(B)$ & Wald & OR & CI (95\%) \\
\hline \multicolumn{6}{|l|}{ Step 1} \\
\hline Sex & -0.397 & 0.245 & 2.62 & 0.67 & $0.42-1.09$ \\
\hline Employment & 0.058 & 0.231 & 0.06 & 1.06 & $0.67-1.67$ \\
\hline Alcohol consumption & -0.004 & 0.011 & 0.11 & 1.00 & $0.97-1.02$ \\
\hline Dependence severity & -0.007 & 0.012 & 0.39 & 1.00 & $0.97-1.02$ \\
\hline $\begin{array}{l}\text { Severity of psychiatric } \\
\text { symptoms }\end{array}$ & 0.000 & 0.007 & 0.01 & 1.00 & $0.99-1.02$ \\
\hline $\begin{array}{l}\text { Alcohol-related } \\
\text { self-efficacy }\end{array}$ & 0.403 & 0.149 & $7.28 *$ & 1.50 & $1.12-2.00$ \\
\hline \multicolumn{6}{|l|}{ Step 2} \\
\hline Taking Steps & 0.059 & 0.027 & $4.77 \dagger$ & 1.06 & $1.01-1.12$ \\
\hline
\end{tabular}

TABLE 3. Results of the Stepwise Regression Analysis With the Number of Standard Drinks on a Typical Drinking Day as the Dependent Variable

\begin{tabular}{lrrrr}
\hline Predictors & $\boldsymbol{\beta}$ & $\mathbf{S E}(\boldsymbol{\beta})$ & $\boldsymbol{T}$ & $\begin{array}{c}\mathbf{R}^{\mathbf{2}} / \mathbf{R}^{\mathbf{2}} \\
\mathbf{C h a n g e}\end{array}$ \\
\hline Step 1 & -0.033 & 0.856 & 0.62 & \\
$\quad$ Sex & -0.120 & 0.824 & $2.27^{*}$ & \\
Employment & 0.070 & 0.040 & 1.24 & \\
$\quad$ Alcohol consumption & 0.065 & 0.042 & 1.14 & \\
$\quad$ Dependence severity & -0.017 & 0.026 & 0.33 & \\
$\quad$ Severity of psychiatric & & & & \\
$\quad$ symptoms & -0.138 & 0.455 & $2.70 \dagger$ & $0.052 \dagger$ \\
$\quad$ Alcohol-related & & & & \\
$\quad$ self-efficacy & & & & \\
Step 2 & -0.147 & 0.093 & $2.83 \dagger$ & $0.073 \dagger$ \\
$\quad$ Taking Steps & & & & \\
\hline
\end{tabular}

$* P<0.05 ; \dagger P<0.01$

$\beta$, standardized regression coefficient; $\mathrm{SE}$, standard error; T, T-value; $\mathrm{R}^{2}$, coefficient of determination.

other predictors were controlled for (Recognition: $B=-0.045$, $P=0.50$; Ambivalence: $B=-0.079, P=0.22$; Taking Steps: $B=-0.095, P=0.14)$.

\section{DISCUSSION}

We examined the contribution of motivation to change substance use (ie, each subscale of the SOCRATES) on drinking outcomes 1 year after discharge from residential alcohol treatment programs. Unlike other studies, we statistically controlled for important predictors of outcomes, such as sex, employment, alcohol consumption before admission, severity of alcohol dependence, severity of psychiatric symptoms at admission, and alcohol-related self-efficacy at discharge. The results suggest that action-oriented motivation to change substance use had a modest impact on drinking outcomes. That is, only the Taking Steps subscale of the SOCRATES was found to be a significant predictor of abstinence and of the number of standard drinks at 1-year follow-up.

Alcohol-related self-efficacy and the Taking Steps subscale were the only 2 significant predictors of abstinence at 1-year follow-up (after controlling for other important 
predictors, including alcohol-related self-efficacy). The other 2 subscales of the SOCRATES (ie, Recognition and Ambivalence) were not associated with abstinence. This suggests that action-oriented motivation has a modest impact on the most important drinking outcome for patients with alcohol dependence. Demmel et al. (2004) also found a modest correlation between 2 SOCRATES subscales and drinking outcomes. In their study, the Taking Steps and Recognition scores accounted for $7.5 \%$ and $1.9 \%$, respectively, of the variance in treatment outcome (ie, abstinence vs relapse).

Alcohol-related self-efficacy more strongly predicted abstinence at 1-year follow-up than did Taking Steps. Similarly, Ludwig et al. (2013) found that alcohol-related selfefficacy in remaining abstinent assessed at discharge from residential AUD treatment was strongly associated with drinking outcome at 1-year follow-up. The abstinence rate was $28.4 \%$ higher in patients with very high alcohol-related self-efficacy than for patients with lower self-efficacy. Alcohol-related selfefficacy at the end of residential treatment might clinically be of more importance in the prediction of drinking outcomes than would the patient's motivation to change substance use at beginning of residential treatment (Adamson et al., 2009). However, Burling et al. (1989) found that self-efficacy at the beginning or end of residential SUD treatment was not associated with abstinence at 6-month follow-up; rather, greater changes from low to high self-efficacy during treatment were related to higher abstinence rates. This suggests that the magnitude of change is more important than high self-efficacy at the end of treatment. Accordingly, it may be useful to improve action-oriented motivation to change substance use during treatment.

Alcohol-related self-efficacy and Taking Steps (controlled for other predictors) were also significantly associated with the number of standard drinks on a typical drinking day at 1 -year follow-up. Again, the 2 other SOCRATES subscales were not significant predictors of this outcome. This result is similar to the findings of Small et al. (2012) that individuals in an outpatient sample with higher scores on the Taking Steps subscale drank less at 12-month follow-up, but those with high Recognition scores drank more. However, consistent with our results, Maisto et al. (2011) found that Recognition scores were not predictive of alcohol consumption. In contrast to our findings, Isenhart (1997) found no significant relationship between the 3 pretreatment readiness for change scores assessed by the SOCRATES and the quantity and frequency of drinking after treatment. However, participants in that study were men, mostly unemployed, and had a shorter treatment duration (21 vs 122 days), which may account for the discrepancy between our results and theirs. Employment seems to be an important predictor variable: in our study, unemployed patients reported consuming more standard drinks at 1-year follow-up than did employed patients.

Overall, patients' acknowledgment of or ambivalence about their alcohol dependence does not seem to be related to drinking status 1 year after treatment, but patients' actions to overcome drinking behavior are related to drinking status (eg, abstinence and the number of standard drinks consumed). Therefore, it is important not only to further examine the association between motivation and abstinence but also to use the number of standard drinks as an important outcome measure. Today, decreasing the amount of alcohol consumed so as to reduce risky drinking is a treatment goal that is accepted by many clinicians and some of the most influential agencies such as the National Institute on Alcohol Abuse and Alcoholism in the United States and the European Medicine Agency (van Amsterdam and van den Brink, 2013).

Time to the first drink did not significantly differ with respect to scores on any SOCRATES subscale. McMahon and Jones (1993) also examined the correlation between time to first drink and motivation assessed by the Readiness to Change Questionnaire (Rollnick et al., 1992) and the Negative Alcohol Expectancy Questionnaire. Their results showed that both the Readiness to Change Questionnaire and the Negative Alcohol Expectancy Questionnaire were valid predictors of treatment outcome and were not correlated with each other (McMahon and Jones, 1996). The inconsistency between this finding and ours may be due to the use of different assessment scales that may measure different components of motivation. The Negative Alcohol Expectancy Questionnaire, for example, was created to measure negative alcohol expectancies, which is an important component of motivation that is not sufficiently assessed by the SOCRATES.

Moreover, researchers have questioned the TTM, which is theoretical substructure of the SOCRATES, because many empirical studies have failed to support the concept of discrete stages of change (Sutton, 2001; West, 2005). West (2005) proposes that when assessing motivations for behavioral change, it is preferable to revert to simple questions about the desire to change, such as those formulated in the Taking Steps subscale. Our results support this view. In addition, West (2006) states that behavioral change in substance use depends only partly on a rational, informed choice based on a stable preference, as suggested by the TTM, and is essentially affected by the individual's external environment (eg, cues and opportunities) and internal processes (eg, habits, impulses, self-control, and identity).

Some limitations have to be considered when interpreting the results of this study. First, the data were based on self-report rather than reports from collaterals or biochemical markers of alcohol use. Del Boca and Darkes (2003) demonstrated that self-report measures, which are relatively inexpensive, noninvasive, and acceptable to respondents, have reasonable levels of reliability and validity. This is particularly true when patients are assessed by research staff not associated with the treatment program, as was the case here. However, in future research, it would be interesting to include objective measures of alcohol consumption. Second, the analyses did not take into consideration the fact that patients were nested within the 12 treatment programs. The patient sample seems to be homogenous, at least for the most important variable, the SOCRATES subscales. However, we do not have information about heterogeneity or homogeneity of other relevant variables (eg, employment). Third, our results are based on a sample from representative Swiss residential AUD treatment programs and might not be generalizable to patients participating in programs in other countries or outpatient and residential programs of different lengths and/or treatment orientations. 


\section{CONCLUSIONS}

Our results suggest that action-oriented motivation to change substance use behavior (ie, the Taking Steps subscale of the SOCRATES) is related to drinking outcomes, in particular, abstinence and the number of standard drinks, at 1-year follow-up. However, the impact of action-oriented motivation at treatment admission is modest when other important predictors of drinking outcome are statistically controlled for. Patients' level of motivation at discharge from residential treatment might be of greater clinical importance than alcoholrelated self-efficacy; consequently, it may be beneficial to focus on improving action-oriented motivation during treatment to change substance use behavior. Future research should focus on which components of motivation at which points in time of treatment are associated with drinking outcomes, and how to improve motivation while patients participate in residential treatment.

\section{ACKNOWLEDGMENTS}

The authors thank all patients and staff members of the residential treatment programs who participated in this study.

\section{REFERENCES}

Adamson SJ, Sellman JD, Frampton CM. Patient predictors of alcohol treatment outcome: a systematic review. J Subst Abuse Treat 2009;36: 75-86.

Bertholet N, Cheng DM, Palfai TP, et al. Does readiness to change predict subsequent alcohol consumption in medical inpatients with unhealthy alcohol use? Addict Behav 2009;34:636-640.

Bertholet N, Cheng DM, Palfai TP, et al. Factors associated with favorable drinking outcome 12 months after hospitalization in a prospective cohort study of inpatients with unhealthy alcohol use. $J$ Gen Intern Med 2010;25:1024-1029.

Burling TA, Reilly PM, Moltzen JO, et al. Self-efficacy and relapse among inpatient drug and alcohol abusers: a predictor of outcome. J Stud Alcohol 1989;50:354-360.

Del Boca FK, Darkes J. The validity of self-reports of alcohol consumption: state of science and challenges for research. Addiction 2003;98:1-12.

Demmel R, Beck B, Richter D, et al. Readiness to change in a clinical sample of problem drinkers: relation to alcohol use, self-efficacy, and treatment outcome. Eur Addict Res 2004;10:133-138.

Derogatis LR. Brief Symptom Inventory. 3rd ed. Minneapolis, MN: National Computer Systems, 1993.

Dilling H, Mombour W, Schmidt MH. Internationale Klassifikation psychischer Störungen. ICD-10 Kapitel V. Klinisch-diagnostische Leitlinien [World Health Organisation (WHO). Tenth Revision of the International Classification of Diseases, Chapter V (F): Mental and Behavioural Disorders. Clinical Descriptions and Diagnostic Guidelines]. Bern, Switzerland: Huber, 1991.

Heather N, McCambridge J. Post-treatment stage of change predicts 12-month outcome of treatment for alcohol problems. Alcohol Alcohol 2013;48:329336.

Hewes RL, Janikowski TP. Readiness for change and treatment outcome among individuals with alcohol dependency. Rehabil Couns Bull 1998; 42:76-93.

Hubard RL, Marsden ME, Cavanaugh JV, et al. Drug and Abuse Treatment. A National Study of Effectiveness. Chapel Hill, NC: University of North Carolina Press, 1989.
Hunter-Reel D, McCrady BS, Hildebrandt T, et al. Indirect effect of social support for drinking on drinking outcomes: the role of motivation. J Stud Alcohol Drugs 2010;71:930-937.

Isenhart CE. Pretreatment readiness for change in male alcohol dependent subjects: predictors of one-year follow-up status. J Studies Alcohol 1997;58:351-357.

Lemke S, Moos RH. Outcomes at 1 and 5 years for older patients with alcohol use disorders. J Subst Abuse Treat 2003;24:43-50.

Ludwig F, Tadayon-Manssuri E, Strik W, et al. Self-efficacy as a predictor of outcome after residential treatment programs for alcohol dependence: simply ask the patient one question! Alcohol Clin Exp Res 2013;37:663667.

Maisto SA, Krenek M, Chung T, et al. A comparison of the concurrent and predictive validity of three measures of readiness to change alcohol use in a clinical sample of adolescents. Psychol Assess 2011;23:983-994.

Project MATCH. Matching alcoholism treatments to client heterogeneity: Project MATCH three-year drinking outcomes. Alcohol Clin Exp Res 1998;22:1300-1311.

Maude-Griffin PM, Finney JW, Moos RH. Multi-site Evaluation of VA Inpatient Substance Abuse Treatment. Measures and Conceptual Domains. Palo Alto, CA: Program Evaluation and Resource Center and HSR\&D Center for Health Care Evaluation VA Palo Alto Health Care System, 1992.

McMahon J, Jones BT. The Negative Alcohol Expectancy Questionnaire. J Assoc Nurse Subst 1993;12:173-186.

McMahon J, Jones BT. Post-treatment abstinence survivorship and motivation for recovery: the predictive validity of the Readiness to Change (RCQ) and Negative Alcohol Expectancy (NAEQ) Questionnaires. Addict Res 1996;4:161-176.

Miller PJ, Ross SM, Emmerson RY, et al. Self-efficacy in alcoholics: clinical validation of the situational confidence questionnaire. Addict Behav 1989;14:217-224.

Miller WR, Tonnigan JS. Assessing drinker's motivation for change: the Stages of Change Readiness and Treatment Eagerness Scale (SOCRATES). Psychol Addict Behav 1996;10:81-89.

Moggi F, Giovanoli A, Buri C, et al. Patients with substance use and personality disorders: a comparison of patient characteristics, treatment process, and outcomes in Swiss and U.S. substance use disorder programs. Am J Drug Alcohol Abuse 2010;36:66-72.

Moggi F, Giovanoli A, Strik W, et al. Substance use disorder treatment programs in Switzerland and the USA: program characteristics and 1-year outcomes. Drug Alcohol Depend 2007;86:75-83.

Moos RH, Cronkite RC, Finney JW. Health and Daily Living Form Manual. 2nd ed. Palo Alto, CA: Mind Garden, 1990.

Ouimette PC, Finney JW, Moos RH. Twelve-step and cognitive-behavioral treatment for substance abuse: a comparison of treatment effectiveness. J Consult Clin Psych 1997;65:230-240.

Prochaska J, DiClemente C. Stages and process of self-change of smoking: towards an integrative model of change. J Consult Clin Psych 1983;51:390395.

Rehm J. The risks associated with alcohol use and alcoholism. Alcohol Res Health 2011;34:135-143.

Rollnick S, Heather N, Gold R, et al. Development of a short 'readiness to change' questionnaire for use in brief, opportunistic interventions among excessive drinkers. Br J Addict 1992;87:743-754.

Small J, Ounpraseuth S, Curran GM, et al. Motivation to change as a mediator for the longitudinal relationships of gender and alcohol severity with oneyear drinking outcome. J Stud Alcohol Drugs 2012;73:504-513.

Sutton S. Back to the drawing board? A review of applications of the transtheoretical model to substance use. Addiction 2001;96:175-186.

van Amsterdam JV, van den Brink W. Reduced-risk drinking as a viable treatment goal in problematic alcohol use and alcohol dependence. J Psychopharmacol 2013;27:987-997.

West R. Time for a change: putting the transtheoretical (stages of change) model to rest. Addiction 2005;100:1036-1039.

West R. Theory of Addiction. Oxford, England: Blackwell Publishing, 2006. 